\title{
RADIAL BASIS FUNCTION DECISION FEEDBACK EQUALISER ASSISTED BURST-BY-BURST ADAPTIVE MODULATION
}

\author{
M. S. Yee, L. Hanzo \\ Dept. of Electr. and Comp. Sc., Univ. of Southampton, SO17 1BJ, UK. \\ Tel: +44-703-593 125, Fax: +44-703-594 508 \\ Email: lh@ecs.soton.ac.uk, http://www-mobile.ecs.soton.ac.uk
}

\begin{abstract}
The performance of radial basis function decision feedback equalised burst-by-burst adaptive modulation is presented for transmission over dispersive wideband mobile channels. Radial Basis Function (RBF) network based channel equalisers have a close relationship with Bayesian schemes. The RBF decision feedback equaliser (RBF DFE) is capable of estimating the 'short term bit error rate' of the received data burst and this estimate is used as the modem mode switching criterion in order to switch between different modulation schemes. This scheme is shown to give a significant improvement in terms of mean bit error rate (BER) and bits per symbol (BPS) performance compared to that of the individual fixed modulation schemes.
\end{abstract}

\section{BACKGROUND}

Burst-by-burst Adaptive Quadrature Amplitude Modulation (AQAM) schemes employ a higher-order modulation scheme, when the channel quality is favourable, in order to increase the throughput and conversely, a more robust lower order modulation scheme is utilized to improve the mean BER performance, when the channel envelope encounters a deep fade. The principles of AQAM scheme were summarized for example by Wong [1] et al. In this paper, we present a novel adaptive modem scheme for transmissions over wideband mobile channels, which employs a Radial Basis Function (RBF) based channel equaliser with decision feedback, in order to mitigate the effects of the dispersive wideband channel. Chen, McLaughlin, Mulgrew and Grant [2] proposed a range of so-called RBF network based channel equalisers, which are potentially capable of errorfreely detecting the received signalling symbols even in a scenario, where the phasors become linearly non-separable due to the inter-symbol interference (ISI) inflicted by the channel. In this situation conventional equalisers would be unable to remove the effects of ISI and hence would exhibit a residual BER. Additionally, Chen et al. [3] introduced decision feedback in their RBF-based equaliser, in order to reduce its computational complexity. The RBF decision feedback equaliser (RBF DFE) was then extended to higherorder QAM schemes, which were investigated in [5]. The

The financial support of the following organisations is gratefully acknowledged: Motorola ECID, Swindon, UK; European Community, Brussels, Belgium; Engineering and Physical Sciences Research Council, Swindon, UK; Mobile Virtual Centre of Excellence, UK. reader is referred to the above references for background reading.

\section{SYSTEM OVERVIEW}

The structure of the joint AQAM and RBF network based equalisation scheme is portrayed in Figure 1. The receiver first extracts the modulation mode used in its received data burst. At the same time the corrupted received data burst is equalised, the BER of the transmitted burst is calculated at the output of the RBF DFE and it is used as the AQAM modem mode switching criterion in order to determine the modem mode to be used during the next transmission burst. This regime assumes a Time Division Duplex (TDD) arrangement, where the uplink (UL) channel quality is estimated on the basis of the Downlink (DL) channel quality.

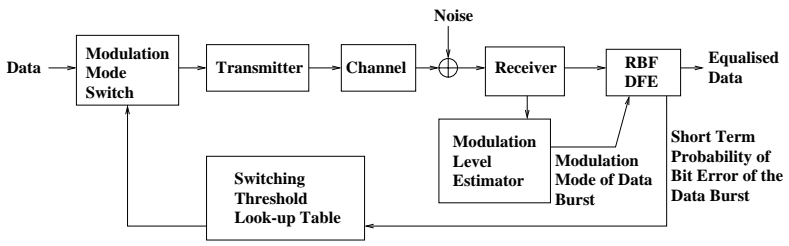

Figure 1: System schematic of the joint adaptive modulation and RBF equaliser scheme

\subsection{Switching Criterion}

The $\mathcal{M}$-QAM RBF equaliser of [5] consists of $\mathcal{M}$ RBF networks that provide the conditional probability density function of each legitimate QAM symbol, $\mathcal{I}_{i}, i=1, \ldots, \mathcal{M}$, if the channel impulse response (CIR) is known. Furthermore, the centers of the RBF network are assigned the values of the channel states. The outputs of the RBF networks are given by $[2,3,5]$ :

$$
\begin{aligned}
\zeta_{i}\left(\mathbf{v}_{k}\right)= & \sum_{j=1}^{n_{s}} w_{j} \varphi_{j}\left(\mathbf{v}_{k}\right) \\
\varphi_{j}\left(\mathbf{v}_{k}\right)= & \exp \left(-\left\|\mathbf{v}_{k}-\mathbf{c}_{j}\right\|^{2} / 2 \sigma^{2}\right) \\
& i=1, \ldots, \mathcal{M}
\end{aligned}
$$

where $\sigma$ is the RBF width, $w_{j}$ are the RBF weights, $\mathbf{c}_{j}$ are the RBF centers and $n_{s}$ is the number of the hidden $\mathrm{RBF}$ nodes, which are represented by $\varphi_{j}$. The a-posteriori 
probability of each legitimate $\mathcal{M}$-QAM symbol, $\varsigma_{i}(k), i=$ $1, \ldots, \mathcal{M}$ can be evaluated from the conditional density function, $\zeta_{i}$ of Equation 1 as follows:

$$
\begin{aligned}
\varsigma_{i}(k) & =P\left(I_{k-\tau}=\mathcal{I}_{i} \mid \mathbf{v}_{k}\right) \\
& =\frac{\zeta_{i}\left(\mathbf{v}_{k}\right) P\left(I_{k-\tau}=\mathcal{I}_{i}\right)}{P\left(\mathbf{v}_{k}\right)} .
\end{aligned}
$$

Therefore - from the a-posteriori probabilities of symbols we can obtain the a-posteriori probabilities of the bits representing the QAM symbols. The a-posteriori probability of the $i$ th bit of the QAM symbols being 0 , for example, is the sum of the a-posteriori probabilities of the symbols with their $i$ th bit being 0 . Therefore, the probability of bit error associated with the decision that the $i$ th bit has the value (either 0 or 1 ) exhibiting the maximum a-posteriori probability, $\tilde{\varsigma}_{\text {bit }}^{i}(k)$, is given by $P_{b}^{i}(k)=1-\tilde{\varsigma}_{\text {bit }}^{i}(k)$. Given the probability of bit error, $P_{b}^{i}(k)$, for the $i$ th bit of an AQAM symbol, the average probability of bit error for a detected AQAM symbol at signalling instant $k$ is given by

$$
P_{b}(k)=\frac{\sum_{i=0}^{\mathrm{BPS}-1} P_{b}^{i}(k)}{\mathrm{BPS}},
$$

where BPS is the number of bits per AQAM symbol and the overall probability of bit error for the detector is given by $P_{\text {bit }}=E\left\{P_{b}(k)\right\}$.

For our joint AQAM and RBF DFE scheme, we are unable to employ the true average probability of bit error $P_{\text {bit }}$ as the modem mode switching criterion, since a prompt burst-by-burst based modem mode selection is required. Hence we invoked the estimated short term probability of bit error or BER, $P_{\text {bit, short-term, which was }}$ estimated by averaging $P_{b}(k)$ of Equation 3 over a single transmission burst according to:

$$
P_{\text {bit }, \text { short-term }}=\frac{\sum_{n=1}^{\mathcal{F}} P_{b}(n)}{\mathcal{F}}
$$

where $\mathcal{F}$ is the number of AQAM symbols per frame. Thus, we could estimate the channel quality on a frame-by-frame basis, based on the estimated $P_{\text {bit, short-term value. }}$

The estimated short-term BER perceived by the receiver is compared to a set of switching BER values corresponding to the various legitimate modulation modes and the modulation mode is selected for the next transmission burst assuming reciprocity of the uplink and downlink. This implies that the similarity of the short-term BER of consecutive UL and DL data bursts can be exploited, in order to set the next modulation mode. The modulation modes utilized in our system are BPSK, 4-QAM, 16-QAM, 64-QAM and no transmission (NO TX).

Therefore, the modulation mode is switched according to the estimated short-term BER, $P_{\text {bit, short-term, as fol- }}$ lows:

$$
\text { Mod. }= \begin{cases}N O T X & \text { if } P_{\mathrm{bit}} \text {, short-term } \geq P_{2}^{\mathcal{M}} \\ B P S K & \text { if } P_{2}^{\mathcal{M}}>P_{\mathrm{bit}} \text {, short-term } \geq P_{4}^{\mathcal{M}} \\ 4 Q A M & \text { if } P_{4}^{\mathcal{M}}>P_{\mathrm{bit}} \text {, short-term } \geq P_{16}^{\mathcal{M}} \\ 16 Q A M & \text { if } P_{16}^{\mathcal{M}}>P_{\mathrm{bit}} \text {, short-term } \geq P_{64}^{\mathcal{M}} \\ 64 Q A M & \text { if } P_{64}^{\mathcal{M}}>P_{\mathrm{bit}} \text {, short-term }\end{cases}
$$

where $P_{i}^{\mathcal{M}}, i=2,4,16,64$ are the switching BER thresholds corresponding to the various $\mathcal{M}$-QAM modes.

\section{SIMULATION RESULTS}

\subsection{Assumptions}

In our simulations the following assumptions were stipulated:

1. Perfect channel impulse response estimation or channel state estimation was used at the receiver. The RBFs' centers were assigned the values of the channel states [5].

2. The channel impulse response is time-invariant for the duration of the transmission burst, but varies from burst to burst, which corresponds to assuming that the channel is slowly varying.

3. We assumed furthermore that the receiver had perfect, ie error-free knowledge of the modulation mode used in its received transmission burst.

4. The RBF DFE used in the system neglected error propagation due to erroneous decision feedback, which in practical terms implied a negligibly low probability of symbol errors, hence feeding the correct symbol to the RBF subset center selection process [5].

\subsection{Simulation Model and BER-motivated Mode Switching}

In our experiments pseudo-random symbols were transmitted in a fixed-length burst. The simulation parameters are listed in Table 1, noting that we analysed the joint AQAM and RBF equaliser scheme over a two-path equal-weight Rayleigh fading channel. The wideband fading channel was frame-invariant. The RBF DFE used in our simulations had a feedforward order of $m=2$, feedback order of $n=1$ and decision delay of $\tau=1$. We will analyse the performance of the joint AQAM/RBF DFE for two systems, a higher integrity scheme, having a target BER of $10^{-4}$, which can be rendered error-free by error correction coding and hence we referred to this arrangement as a data transmission scheme; the lower integrity scheme was designed for maintaining a BER of $10^{-2}$, which is adequate for speech transmission especially in conjunction with FEC. The target BPS values of these schemes were 3 and 4.5 bits per symbol, respectively.

\begin{tabular}{|l|l|}
\hline No. of data symbols per burst & 144 \\
No. of training symbols per burst & 27 \\
Transmission Frequency & $1.9 \mathrm{GHz}$ \\
Transmission Rate & $2.6 \mathrm{MBd}$ \\
Vehicular Speed & $30 \mathrm{mph}$ \\
Normalised Doppler Frequency & $3.3 \times 10^{-5}$ \\
Channel weights & $0.707+0.707 z^{-1}$ \\
\hline
\end{tabular}

Table 1: Simulation parameters

The BER switching thresholds corresponding to $\mathcal{M}$ QAM, $P_{i}^{\mathcal{M}}, i=2,4,16,64$, can be obtained by estimating the BER degradation/improvement, when the modulation mode is switched from $\mathcal{M}$-QAM to a higher/lower number of modulation levels. In this experiment, we obtain this BER degradation/improvement measure from the estimated short-term BER of every modulation mode used, under the same instantaneous channel conditions. Figure 2 shows the estimated short-term BER of all the possible 


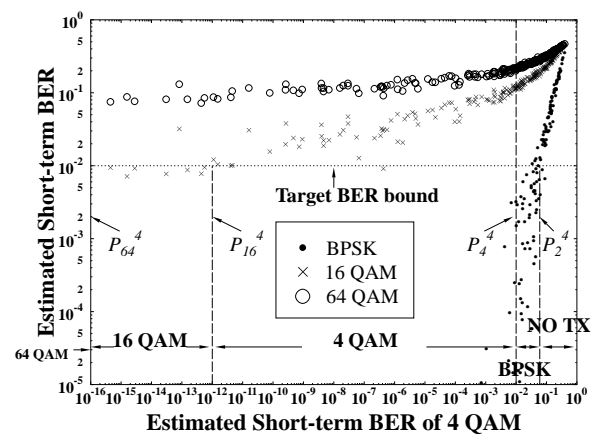

Figure 2: The estimated short-term BER for all the possible modulation modes that can be invoked, assuming that the current mode is 4-QAM - versus the estimated short-term BER of 4-QAM for the two-path Rayleigh fading channel of Table 1 .

modulation modes that can be invoked, assuming that the current mode is 4-QAM, versus the estimated short-term BER of 4-QAM under the same instantaneous channel conditions. The short-term BERs of the modulation modes are obtained on a burst-by-burst basis from Equation 4. In order to obtain the target BER of $10^{-2}$, Figure 2 demonstrates how each switching BER threshold $P_{i}^{4}$ is obtained. For example, if the estimated short-term BER of the received 4QAM transmission burst is below $P_{16}^{4}=10^{-12}$, the modulation mode can be switched to 16-QAM for the next transmission burst, since the short-term BER of this 16QAM transmission burst is expected to be below the target BER of $10^{-2}$. The 4QAM error probability of $P_{16}^{4}=10^{-12}$ used in this example for switching to 16QAM appears extremely conservative, but it is justified by the large uncertainty associated with the estimation of the BER due to the Rayleigh-faded impulse response taps. This manifests itself also in the rather spread nature of the BER estimates in Figure 2. A feasible technique for mitigating this phenomenon is employing the fade-tracking scheme of Figure 11.2 in Reference [6].

Using the method demonstrated by Figure 2, the switching BER thresholds were obtained for the target BER of $10^{-2}$ and $10^{-4}$, as listed in Table 2 and Table 3 , respectively in the context of all possible combinations of the mode transitions. Note that the extremely low values for $P_{16}^{4}=1 \times 10^{-45}$ and $P_{64}^{16}=1 \times 10^{-50}$ in Table 3 were obtained by extrapolating the curves similar to Figure 2 but for 16-QAM and 64-QAM, respectively, in order to achieve the target BER of $10^{-4}$.

\subsection{RBF/AQAM Performance Comparison with Constituent Fixed Modulation Schemes}

The performance of the joint AQAM/RBF DFE is compared with that of the constituent fixed modulation schemes in Figure 3. The BER and BPS performance was evaluated for two different adaptive modulation schemes. In the first scheme, the transmitter always transmitted data without transmission blocking or disabling. By contrast, in

\begin{tabular}{|c|c|c|c|c|}
\hline & $P_{2}^{\mathcal{M}}$ & $P_{4}^{\mathcal{M}}$ & $P_{16}^{\mathcal{M}}$ & $P_{64}^{\mathcal{M}}$ \\
\hline NO TX & $9 \times 10^{-3}$ & $5 \times 10^{-5}$ & 0.0 & 0.0 \\
\hline BPSK & $1 \times 10^{-2}$ & $5 \times 10^{-5}$ & 0.0 & 0.0 \\
\hline 4-QAM & $6 \times 10^{-2}$ & $1 \times 10^{-2}$ & $1 \times 10^{-12}$ & 0.0 \\
\hline 16-QAM & $2 \times 10^{-1}$ & $1 \times 10^{-1}$ & $1 \times 10^{-2}$ & $1 \times 10^{-8}$ \\
\hline 64-QAM & $3 \times 10^{-1}$ & $2 \times 10^{-1}$ & $9 \times 10^{-2}$ & $1 \times 10^{-2}$ \\
\hline
\end{tabular}

Table 2: The switching BER thresholds $P_{i}^{\mathcal{M}}$ of the joint adaptive modulation and RBF DFE scheme for the target BER of $10^{-2}$ over the two-path Rayleigh fading channel of Table 1.

\begin{tabular}{|c|c|c|c|c|}
\hline & $P_{2}^{\mathcal{M}}$ & $P_{4}^{\mathcal{M}}$ & $P_{16}^{\mathcal{M}}$ & $P_{64}^{\mathcal{M}}$ \\
\hline NO TX & $9 \times 10^{-5}$ & $1 \times 10^{-15}$ & 0.0 & 0.0 \\
\hline BPSK & $1 \times 10^{-4}$ & $1 \times 10^{-15}$ & 0.0 & 0.0 \\
\hline 4QAM & $1.5 \times 10^{-2}$ & $1 \times 10^{-4}$ & $1 \times 10^{-45}$ & 0.0 \\
\hline 16QAM & $1.2 \times 10^{-1}$ & $5 \times 10^{-2}$ & $1 \times 10^{-4}$ & $1 \times 10^{-50}$ \\
\hline 64QAM & $2.2 \times 10^{-1}$ & $1.5 \times 10^{-1}$ & $3 \times 10^{-2}$ & $1 \times 10^{-4}$ \\
\hline
\end{tabular}

Table 3: The switching BER thresholds $P_{i}^{\mathcal{M}}$ of the joint adaptive modulation and RBF DFE scheme for the target BER of $10^{-4}$ over the two-path Rayleigh fading channel of Table 1.

the second scheme, dummy data was transmitted, whenever the estimated short-term BER of the received BPSK transmission burst was higher than the target BER, a scenario, which we refered to as transmission blocking. The transmission of dummy data during blocking allowed us to keep monitoring the BER, in order to determine when to commence transmission and in which modem mode.

We will commence by analysing Figure 3(a), where the joint AQAM/RBF DFE scheme was designed for speech transmision, i.e. for a BER of $10^{-2}$. For the adaptive scheme, which did not incorporate transmission blocking, the performance with adaptive modulation was better or equivalent to the performance of BPSK in terms of the mean BER and mean BPS for the SNR range between $0 \mathrm{~dB}$ and $8 \mathrm{~dB}$. At the channel SNR of $8 \mathrm{~dB}$, even though the mean BER performance is equivalent for the BPSK mode of the adaptive scheme and for the fixed-mode BPSK scheme, the mean BPS for the adaptive scheme improved by a factor of 1.35, resulting in a mean BPS of 1.35. In the SNR range of $8 \mathrm{~dB}$ to $15 \mathrm{~dB}$, the adaptive scheme outperformed the $4 \mathrm{QAM}$ scheme in terms of its mean BER performance. At the channel SNR of $15 \mathrm{~dB}$, the mean BERs of both schemes are equivalent, although the mean BPS of the adaptive scheme is approximately 2.6, resulting in a substantial improvement, when compared to 4QAM. The adaptive scheme that utilized transmission blocking achieved a mean BER below $10^{-2}$. As the SNR improved, the performance of the adaptive schemes both with and without transmission blocking converged, since the probability of encountering high shortterm BERs reduced. The mean BER and mean BPS performance of both adaptive schemes converged to that of 64QAM for high SNRs, where 64QAM became the dominant modulation mode.

Similar trends were observed for data-quality transmission, i.e. for the $10^{-4}$ target BER scheme in Figure 3(b). 
However, we note that for the SNR range between $8 \mathrm{~dB}$ to $17 \mathrm{~dB}$, the mean BER of the adaptive scheme without transmission blocking was better, than that of BPSK. This phenomenon was also observed in the wideband joint AQAM and DFE scheme of [1], which can be explained as follows. The mean BER of the system is the ratio of the total number of bit errors to the total number of bits transmitted. The mean BER will decrease with a decreasing number of bit errors and with an increasing number of the total bits transmitted in the data burst. For a fixed number of symbols transmitted, the number of total bits transmitted in a data burst is constant for the BPSK scheme, while for the adaptive scheme the total number of bits transmitted in a data burst increased, when a higher-order modulation mode was used. However, when a higher-order modulation mode was used for transmission, the probability of erroneous bits increased. If the relative bits per symbol increment upon using AQAM is higher than the relative bit error ratio increment, then the mean BER of the adaptive scheme will be improved. Consequently the adaptive mean BER can be lower than that of BPSK.

In summary, the joint adaptive modulation and RBF DFE scheme has its advantages, when compared to the individual fixed modulation schemes in terms of mean BER and mean BPS performance. Note however that the target performance for speech and data transmission in terms of the mean BPS (4.5 and 3, respectively) can only be achieved for the adaptive scheme with and without transmission blocking, if the channel SNR is above about $22 \mathrm{~dB}$. Thus, the advantage of using an adaptive scheme with transmission blocking is that the performance of the joint AQAM and RBF DFE scheme can be 'tuned' to a certain required mean $B E R$ performance. Furthermore, due to the modem mode switching the associated bit errors exhibit a less bursty error distribution than that of fixed modems, which renders the AQAM scheme more amenable to channel coding, resulting in higher channel coding gains. However, the disadvantage is that the utilization of transmission blocking results in transmission latency - an issue which was addressed in reference [7].

\subsection{Comparison with Upper Bound Performance}

In this section, we compare the performance of the joint AQAM/RBF DFE with its upper bound performance and derive the BER switching thresholds, $P_{i}^{\mathcal{M}}, i=2,4,16,64$. In deriving the upper bound performance of the joint adaptive modulation and RBF based equalisation scheme, in addition to the assumptions listed in Section 3.1, we also assume that the short-term BER estimate $P_{\text {bit, short-term }}$ is known prior to transmission for all the modulation modes used in the system. We also assume that given the esti-

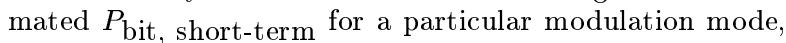
the transmitter has perfect knowledge of the corresponding short-term probability of bit error for the other modulation modes used in the system under the same channel conditions.

In our experiments pseudo-random symbols were transmitted in a fixed-length burst for all modulation modes over the frame-invariant wideband channel. The receiver received each data burst having different modulation modes and equalised each one of them independently. The estimated short-term BER for each modulation mode was ob-

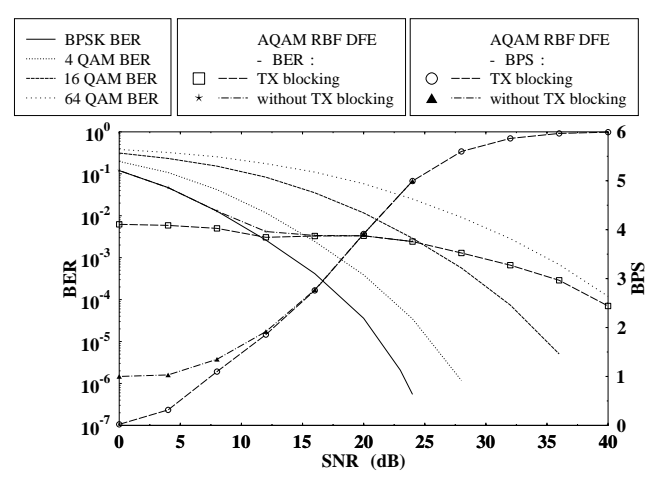

(a) Speech Transmission

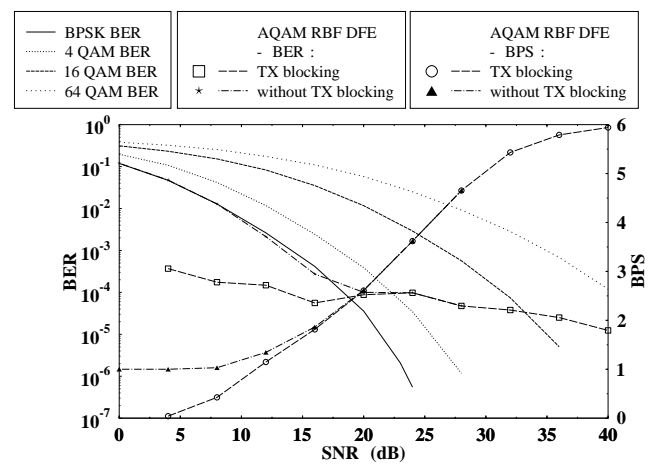

(b) Data Transmission

Figure 3: The BER and BPS performance of the joint AQAM/RBF DFE, showing also the BER performance of the constituent fixed modulation schemes, namely BPSK, 4QAM, 16-QAM and 64-QAM, over the two-path Rayleighfading channel of Table 1 . The modem mode switching levels used for the joint AQAM/RBF DFE scheme are listed in Table 2.

tained. The highest-order modulation mode, $\mathcal{M}^{*}$ that provided a short-term BER $P_{\text {bit, short-term }}^{\mathcal{M}^{*}}$, which was below the target BER $P_{\text {bit, target, when: }}$

$\mathcal{M}^{*}=\max \left\{\begin{array}{l}\mathcal{M}=2,4,16,64, \\ \left.\text { such that } P_{\text {bit, }}^{\mathcal{M}} \text {, short-term } \leq P_{\text {bit, target }}\right\}\end{array}\right.$,

was chosen to be the actual modulation mode that was used by the transmitter and its received equalised frame was used for the actual BER calculation of the system.

Figure 4(a) shows the BER and BPS performance of the joint AQAM/RBF DFE scheme designed for speechtransmission with the switching thresholds given in Table 2 - when using the current received frame's BER estimate, in order to determine the modem mode of the next transmis- 


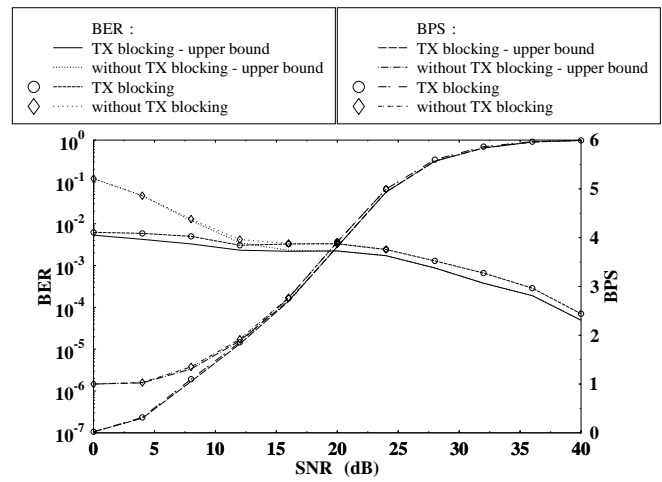

(a) Speech Transmission

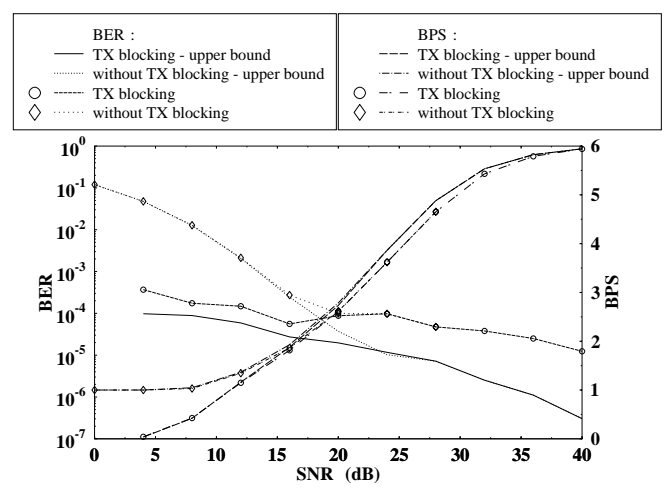

(b) Data Transmission

Figure 4: The BER and BPS performance of the joint AQAM/RBF DFE scheme using the current BER estimate in order to estimate the next burst's transmission mode, and its upperbound performance, using the parameters listed in Table 1 . The modem mode switching levels used for the joint AQAM/RBF DFE scheme are listed in Table 2 and Table 3 for speech-transmission and data-transmission, respectively.

sion burst - in contrast to its upper bound performance. The performance comparison shows that there is little performance degradation, when the current received burst's short-term BER estimate is used to control the modulation mode of the next transmission burst based on the switching parameters of Table 2 for the AQAM scheme designed for speech transmission. Since the channel of Table 1 is slowly varying, the performance of the joint AQAM/RBF DFE scheme based on the switching parameters of Table 2 is comparable to its upper bound performance.

Figure 4(b) shows the BER and BPS performance of the joint AQAM/RBF DFE scheme designed for data-transmission with the switching thresholds given in Table 3 in comparison to its upper bound performance. The degrada- tion with respect to the upper bound performance of the AQAM/RBF DFE scheme designed for data-transmission is more significant compared to the adaptive scheme designed for speech-transmission, as seen in Figure 4(a) and Figure $4(\mathrm{~b})$. Note that for the low BER switching thresholds of $P_{16}^{4}\left(=1 \times 10^{-45}\right)$ and $P_{64}^{16}\left(=1 \times 10^{-50}\right)$ in Table 3 , which were used in the adaptive scheme for the target BER of $10^{-4}$, the RBF DFE is unable to provide a high accuracy BER estimate. As the SNR improves, the frequency of encountering the switching thresholds $P_{16}^{4}$ and $P_{64}^{16}$ increases and thus the performance degradation compared to the upper bound increases.

\section{CONCLUSIONS}

The RBF DFE was shown to provide an 'on-line' BER estimation of the received data burst, which was used as the modem mode switching criterion for the adaptive modulation scheme. Our simulation results showed that the proposed RBF DFE-assisted burst-by-burst adaptive modem outperformed the individual constituent fixed modulation modes in terms of mean BER and BPS performance. A method to obtain the switching BER thresholds of the joint AQAM/RBF DFE scheme was proposed and was shown to provide little performance degradation in comparison to the associated upper bound performance.

\section{REFERENCES}

[1] C. H. Wong and L. Hanzo, "Channel Capacity Upperbound of a Wideband Burst-by-burst Adaptive Modem," Proc. of VTC'99, Houston, USA, pp. 1851-1855, May 1999.

[2] S. Chen, B. Mulgrew, and P. M. Grant, "A clustering technique for digital communications channel equalization using radial basis function networks," IEEE Transactions on Neural Networks, vol. 4, no. 2, pp. 570-579, July 1993.

[3] S. Chen and S. McLaughlin and B. Mulgrew, "Complexvalued radial basis function network, Part II: Application to digital communications channel equalisation," EURASIP Signal Processing, vol. 36, no. 2, pp. 175188, March 1994.

[4] S. Chen and S. McLaughlin and B. Mulgrew and P. M. Grant, "Adaptive Bayesian Decision Feedback Equalizer for Dispersive Mobile Radio Channels," IEEE Transactions on Communications, vol. 43, no. 5, pp. 1937-1945, May 1995.

[5] M. S. Yee and L. Hanzo, "Multi-level radial basis function network based equalisers for rayleigh channels", Proc. of VTC'99, Houston, USA, pp. 707-711, May 1999.

[6] W. T. Webb and L. Hanzo, "Modern Quadrature Amplitude Modulation: Principles and Applications for Fixed and Wireless Channels," London: IEEE - JohnWiley, 1994

[7] J. M. Torrance, L. Hanzo: Latency and Networking Aspects of Adaptive Modems over Slow Indoors Rayleigh Fading Channels, IEEE Tr. on Veh. Techn., July 1999, Vol. 48, No. 4, pp 1237-1251 